\title{
APPL YING MODELING AND SIMULATION TO ENHANCE NATIONAL AND MULTI-NATIONAL COOPERATION
}

\author{
Ronald J. ROLAND
}

\section{Introduction}

This paper is the first in a series that will describe the application of modeling and simulation to enhance country-to-country, agency-to-agency and coalition-tocoalition cooperation and understanding. The goal of the series is to provide information on the availability of $M \& S$ applications, their potential benefits and associated cost, develop a hypothetical plan for the implementation and use of various M\&S applications and, finally, encourage a dialogue of understanding about the benefits and requirements to obtain, implement and execute $M \& S$ tools.

What really generates success in civil/military operations? Many cite technological superiority in weaponry, intelligence of the adversary's intentions or sometimes just raw numbers. Yet the greatest victories and successes in history have usually involved smaller forces defeating larger ones, frequently against odds that no gambler would take. What is the critical edge that allows smaller forces to defeat their more powerful opponents? Part of it is experience, though this is frequently bought at the price of lives and lost opportunities. The truth is that in lieu of tempering units in the forge of battle, the crucible of training is what can make the difference in combat and in civil operations. Training is frequently viewed by some leaders, military and non-military, as a wasteful misuse of funds that might be better used to sustain larger forces or to buy new and more effective equipment. More thoughtful individuals realize, though that often the quality of the operator can overcome the capabilities of better equipment in the hands of poor operators. This is the training philosophy that has driven nations like the United States and the United Kingdom for the last generation, and has allowed so many victories and successes.

Herein we provide a background concerning one effort to proliferate a common architecture for crisis management, a history of $M \& S$ development and an 
introduction to a specific software application that is widely used to train senior staffs within simulated crises situations. The focus shall be on computer aided exercises, training and analysis. It will evolve through a series of articles. The intent is to convince the audience that the use of M\&S will provide them an efficient and effective means to maintain their edge to better manage or perhaps avoid future conflicts.

\section{A National Crisis Management Command Center}

If you think education is expensive, try ignorance.

Unknown teacher

Although the demise of the Warsaw Pact in 1989 has changed the political landscape of the world, natural disasters and other crisis situations continue as hostile threats to all nations. Devastating earthquakes, floods, environmental disasters and a host of other catastrophes may cause devastation with loss of lives and considerable property damage. Illegal activities such as drug trafficking and smuggling also occur at all levels. An important issue is that none of these events recognize political boundaries. In response to these situations, departments, agencies and nations need to effectively apply local, regional, and national resources to manage the consequences of disasters and crises. Increasing in importance is the ability to work across national boundaries to collaborate and inter operate with multi national resources. These resources typically include some combination of civil and military units that may be called upon to provide assistance in the face of crisis situations. ${ }^{1}$

In order for these resources, both civil and military components, to respond to crisis situations in an efficient manner there are at least three fundamental requirements; (1) the availability of information regarding crisis situations and military/civil resources readiness; (2) coordination among the organizations and agencies (intra and international) involved in crisis management; and (3) continued training and exercising of the resources so that they can respond effectively when needed. Herein we address the operational aspects of an information support system intended to assist national Ministries of Defense (MODs) in coordinating with other national (and regional) organizations dealing with crisis situations and also in applying military and civil resources in execution of crisis management responsibilities in coordination with other national and international organizations.

In the course of executing Command, Control, Communications and Computers (C4) studies for Central and Eastern European nations, a common thread emerged from the analysis of national $\mathrm{C} 4$ system requirements and on-going modernization plans. All nations involved in the studies were engaged in planning for the introduction of centralized information collection and processing systems to support the management 
of military forces in crisis situations. Because these systems were being planned independently, there was little commonality of system concepts or system architectures. Consequently, the ability to share information within and among nations in a regional crisis and to collaborate in crisis relief actions would, most likely, be severely limited.

In response to the apparent need for a centralized crisis management capability and in the spirit of the Regional Airspace Initiative, which resulted in an Air Sovereignty Operations Center (ASOC) program, the U.S. Air Force Electronic Systems Center (ESC) developed a concept for implementation of a national command center for crisis management. This command center, identified as the NMCC, would support both national civil and military crisis situations and, because different national systems would be built on a common architectural platform, it would also support regional collaboration in response to regional crisis situations. This NMCC concept was presented to several nations in the spring of 1999 and met with favorable response. The presentation also included a discussion concerning a software component, the Joint Theater Level Simulation (JTLS) to be considered as a baseline for training and analysis at the national and multi-national command or decision making levels. ${ }^{2}$

Based on the initial favorable response, the U.S. government formally introduced the new policy initiative to Partnership for Peace nations at a multinational conference in Sofia, Bulgaria in June 1999. As described in the U.S. keynote address at the conference, the NMCC is intended to provide national command authorities with a modern, integrated command and control center to support decision making in the event of civil or military crises. Further, the NMCC will be built on a NATOcompatible technical architecture platform and will provide interfaces that are compatible with comparable NATO and U.S. command and control systems.

Seven countries at the conference indicated they would collaborate in the initiative, most as active participants. Since that time, several other countries have expressed interest in the initiative. The next step in the process of advancing the initiativeforming a multinational Working Group of potential program participants-occurred in Predeal, Romania in September 1999. This Working Group will modify and agree this Concept of Operations (CONOPS) for the NMCC and also establish consensus on a technical architecture framework for the NMCC. These two documents will serve as the foundation for an acquisition effort for those nations choosing to participate in the NMCC program. 


\section{Modeling and Simulation Support}

The NMCC Concept of Operations (CONOPS) does not include a conceptual model for modeling and simulation $(M \& S)$. As we know, $M \& S$ has been extensively and successfully applied to a wide range of civil/military problems including combat, operations other than war, acquisition, decision making, contingency plan analysis, logistics and communications. ${ }^{3}$ I has been used as a decision support tool to evaluate how a civil/military force should be constituted, how it might be deployed, and how its organic resources or weapon systems should be acquired and maintained. The crisis management regime often requires domain experts because the development and use of simulation models to support an NMCC require specialized knowledge in unique problems. Most military models have been developed by highly specialized groups and used in narrowly focused user communities. For example, the Army is interested in wargaming simulation with ground forces. The Navy is interested in battle group simulation with aircraft carriers, aircraft and ships. The Marine Corps' interest is in amphibious operations, and the U.S. Air Force is working on space systems, strategic, long-range bombing and tactical air-to-air and air-to-ground support. Each service adopts different logistics systems for weapons systems maintenance; the Navy and the Marine Corps follow three levels (organizationallevel, intermediate-level, and depot-level) of maintenance, while the Air Force uses two levels of maintenance, and the Army has five levels. ${ }^{4}$

As a result, there are many organizations and agencies involved in M\&S. Each community uses its own special jargon, abbreviations, and acronyms, which makes it difficult for the various government agencies and services to communicate. More than 150 pages of Glossary of DoD M\&S Terms are available from Department of Defense (DoD) Directive 5000.59-M. ${ }^{5}$ Although the simulation community is huge, there is a lack of systematic communication and no central resource library. Many models have been developed on a stand-alone, system specific, as-needed, and as-afforded basis, which has resulted in redundant investments. Typically, more efforts are spent to develop a new simulation system and building the simulation infrastructure than the efforts to develop components specific to purpose of the simulation. If the infrastructure and other simulation components could be reused, the payoff would be enormous. For this reason, object-oriented programming and the high level architecture (HLA) are getting more attention.

Although M\&S has been used to investigate military problems for many years, there is very little literature available for general readers who do not specialize in military simulation. Military simulation models are different from others because (1) many of them are highly classified with details that could not be widely disseminated; (2) weapon capabilities and use are not typically used in other M\&S; (3) certain algorithms are closely controlled to avoid reverse engineering by potential 
adversaries; and (4) the use of certain equations, e.g., Lanchester, which is often used in wargaming simulation, is not typical of commercial M\&S. The purpose of this paper is to provide the reader an overview of military simulation and insight to its future directions. We review recent developments in military modeling, particularly in wargaming simulation. We provide sources of many DoD documents including internet homepage addresses, where applicable, so that the reader can retrieve the updated information. Since more and more DoD documents are available electronically via the internet, it becomes easier for simulationists to access information on simulation topis. There are tremendous opportunities for expansion of simulation applications in every aspect of life.

\section{Organizations}

The DoD and the Joint Staff maintain their own agencies for M\&S. In addition, each Service maintains M\&S offices. In June 1991, the Defense Modeling and Simulation Office (DMSO) was established by the Under Secretary of Defense for Acquisition and Technology. Two responsibilities of DMSO are to publish DoD M\&S policy and promote cooperation among DoD agencies. In January 1994, the Deputy Secretary of Defense promulgated DoD Directive 5000.59 to the DoD community. This directive is entitled DoD Modeling and Simulation $(M \& S)$ Management, and was a DOD-wide effort to establish policy for M\&S. It was a significant step toward centralizing the management of DoD M\&S activities. In accordance with the DoD Modeling and Simulation Master Plan (DoD 5000.59-P, dated October 1995), DMSO is leading a DoD-wide effort to establish a common technical framework to facilitate the interoperability of all types of models and simulations among themselves and with command, control, communication, computer, and intelligence (C4I) systems, as well as to facilitate the reuse of M\&S components. This Common Technical Framework includes the High Level Architecture (HLA), which represents one of the highest priority efforts within the DoD modeling and simulation community.

The DoD Modeling and Simulation Master Plan initial definition of the M\&S HLA was accomplished under the sponsorship of the Defense Advanced Research Projects Agency (DARPA) Advanced Distributed Simulation (ADS) program. It was transferred to DMSO in March 1995 for further development by the DoD-wide Architecture Management Group (AMG). Central to this task was the development of a set of prototypes that addressed critical issues in the HLA. In September 1996, the Under Secretary of Defense for Acquisition and Technology (USD(A\&T)) approved HLA as the standard technical architecture for all DoD simulations and required all computer simulations for military operations meet the HLA standardization requirements by FY2001. Dr. Kaminski's directive mandated that all DoD 
simulations, failing to comply with HLA standards by a specified date, be retired from service. ${ }^{6}$

The Executive Council for Modeling and Simulation (EXCIMS) ${ }^{7}$ is a high level advisory group on DoD M\&S policy, initiatives, standards, and investments. More details about DoD simulation activities and DoD Directive 5000.59 can be obtained on the DMSO internet homepage.

As was mentioned, each Service maintains its own M\&S activities. The Army has long M\&S history and is better organized than the rest of the services. Deputy Undersecretary of the Army for Operations Research (DUSAOR) oversees all Army Modeling and Simulation. The Army Modeling and Simulation Office (AMSO) ${ }^{8}$ is the operational activity for Army M\&S. The Army maintains the modeling and simulation homepage for the Army Modeling and Simulation Resource Repository (MSRR). ${ }^{9}$ The Army's National Simulation Center ${ }^{10}$ located in Ft. Leavenworth, Kansas, supports simulation training exercises around the world. The Army currently maintains six major simulation models for training. They are Janus, VICTORS (Variable Intensity Computerized Training System), BBS (Brigade/Battalion Battle Simulation), CBS (Corps Battle Simulation), TACSIM (Tactical Simulation) and CSSTSS (Combat Service Support Training Simulation System). The details of these models and a list of other Army simulation models are available in MOdels \& Simulations: Army Integrated Catalog (MOSAIC). ${ }^{11}$

The Air Force also has a long history of M\&S applications. The Directorate for Modeling and Simulation (see http://xoc.hq.af.mil) is the single point of contact in the Air Force for policy on modeling, simulation and analysis activities. It includes the Evaluation Support Division, Technical Support Division, Warfighting Support Division, and Air Force Studies and Analysis Agency.

The Navy and the Marine Corps have smaller M\&S organizations compared to the Army and the Air Force. They have a Modeling and Simulation Advisory Council that guides the development of policy, coordination and technical support and promotes the use of the Navy-wide common support services. The Navy and the Marine Corps maintain their own Modeling and Simulation Management Offices (see http://mcmsmo.usmc.mil), and set their own M\&S policies. A simulation model, the Research, Evaluation, and Systems Analysis (RESA) was developed as a naval warfare command, control and communication (C3) analysis tool for the Navy. The Marine Air Ground Task Force Tactical Warfare Simulation (MTWS) is one of the Marine Corps' tactical combat simulation models. ${ }^{12}$ 


\section{Classification of Military Simulation Models}

According to the Defense Science Board, military simulations are classified into three categories: live, virtual, and constructive. While there is no clear-cut distinction among these categories, it is still helpful to understand the basic differences. Live simulation involves real people and real systems. Operational test and evaluation (OT\&E), and military field exercises are examples. Live simulations in support of training are conducted at the Army National Training Center (NTC) ${ }^{13}$ located in Ft. Irwin, California; the Navy "Strike University" in Fallon Naval Air Station, Nevada; the Air Force Red Flag Site at Nellis Air Force Base, Nevada; and the Marine Corps Air-Ground Combat Center in Twenty Nine Palms, California.

The NTC provides an example of a live simulation. It is a vast expanse of desert approximately the size of the State of Rhode Island. This is where the Army conducts training exercises in order to prepare itself for war in the desert. There are approximately 2,500 soldiers permanently stationed at NTC who function as the "home team." This group pretends to be the enemy and uses all of the doctrine and tactics of the opposing force. The visiting teams arrive at NTC twelve times a year and conduct wargame type simulations against the home team. Every move and every shot fired is monitored by a powerful laser engagement system that records all of the signals from the pieces of armor and other equipment that are participating in the exercise. All of this information is fed into the computer simulation, and numerous statistics are tallied so that, at the end of the exercise, both teams can be evaluated and areas of improvement can be identified.

Virtual simulation involves real people in a simulated system. This includes aircraft and tank simulators. ${ }^{14}$ This type of simulation is helpful in training, and for evaluating control, decision and communications skills. Virtual simulation has become more popular with developments in computer technology, especially computer graphics. The journal Military Simulation \& Training $^{15}$ is a good source for up-to-date information on military training simulators. In constructive simulations, humans may (or may not) interact with the model and everything is simulated. Constructive simulations of combat include wargames for training as well as for analytical tools. Constructive simulations may be used for training events that range from senior staffs to the operator-level. For example, JTLS is a constructive simulation, which can be used for staff training as well as for operations planning analysis. We now describe JTLS in more detail.

\section{The Joint Theater Level Simulation}

The simulation of combat, or a wargame, is used more and more extensively to reduce cost and maintain a trained force. It is an inexpensive alternative to live 
training exercises. Simulations are also very useful for testing and evaluating proposed procedures, strategies and various systems such as economic, weapons communications and civil architectures. We present and discuss the Joint Theater Level Simulation (JTLS) as an illustration of a wargaming simulation. ${ }^{16}$ The purpose is to illustrate various aspects of wargaming simulation using JTLS as an example. JTLS is a theater level simulation that models ground, air and water based resources.

The development of JTLS began in 1983 as a project funded by three Army organizations: the U.S. Readiness Command, the U.S. Army Concepts Analysis Agency, and the U.S. Army War College. ${ }^{17}$ It has had continuous functional and system upgrades since that time. Its primary focus is on conventional joint and combined operations and is currently managed by the U.S. Joint Forces Command/ Joint Warfighting Center, Suffolk, Virginia.

JTLS was designed as a theater-level model for commanders and planners as an Operations Plan (OPLAN) analysis tool, support material for education, command post exercise support for training, and a primary means to investigate the results of combat and civil affairs. It is heavily used as an exercise driver where JTLS provides the environment for the dynamic interactions of intelligence, air, logistics, naval, and ground forces. This environment allows users to develop insight into the relative merits of alternative courses of action, force structures, combat systems, and procedures.

The model is currently in use by numerous agencies including the Joint Warfighting Center, the Warrior Preparation Center, NATO's Command Control and Consultancy Agency (NC3A), the National Defense University, the Army War College, the Naval Postgraduate School, Combined Forces Command Korea, the Australian Defense Force Warfare Centre and the South Korean Institute for Defense Analysis. It is installed at Hellenic National Defense College's M\&S Center, the Defence Evaluation and Research Agency, United Kingdom and the Turkish War College. The Louisiana State University, MITRE Corporation and RAND Corporation have evaluating JTLS for application to non-combatant environments or potential research purposes. ${ }^{18}$

JTLS is a multi-sided, interactive, computer-driven simulation. In this context, multisided really means that there can be up to ten sides depicting various organizations whether friendly, neutral, hostile, unknown and/or civilian. This is a dymanic environmental variable that is set at each instantiation of JTLS. ${ }^{19}$ One recent JTLS scenario includes the sides called the Gulf Coalition, United Nations (UN) Forces, Israel, Iraq and Iran. Each side in turn consists of one of more factions limited by the hardware, scenario requirements and users' imaginations. The Gulf Coalition factions included Saudi Arabia, Kuwait and one that represents the "civilian populace." All of 
the UN members were included as factions within the UN side. Factions are also included within the other sides, which permitted an accurate depiction of the forces and perturbational influences within the Gulf region.

Each JTLS side can be subdivided into an unlimited number of factions. A faction's side allegiance is dynamically changeable during the game (scenario). Side relationship is asymmetric and can also be changed during the game. A large number of players (for example 300) can be involved simultaneously in a single game. The actual number is defined by the User when planning for the training event. Since JTLS can be highly distributed depending on the communications available, the Players can literally be anywhere in the world or all in the same room. In the case of using JTLS as an analysis tool, a meaningful analysis can be managed by as few as two people. JTLS models coalition air, land, sea, amphibious, and special forces operations. The model can support limited nuclear and chemical effects, low intensity conflict, and pre-conflict operations. The model also supports the representation of civilian and non-combatant forces.

The JTLS system consists of six major software modules and numerous smaller support programs that work together to prepare the scenario, run the game, and analyze the results. Designed as a tool for use in the development and analysis of operation plans, the model is theater-independent, that is the data for a specific scenario are stored in a database separate from the source or object code. The database may contain highly classified or sensitive and is purposely maintained independent of the software until execution. The JTLS program itself is unclassified.

Model features include Lanchester attrition algorithms, detailed logistics modeling, and explicit air, ground, and naval force movement. In addition to the model itself, the JTLS system includes software designed to aid in scenario database preparation and verification; entering game orders; and obtaining scenario situational information from graphical map displays, messages, and status displays. The movement of forces within any combat environment is affected by the terrain. The terrain is represented as a hexagonal grid overlay on a map projection. The maximum geographic region or area used in a JTLS scenario is 2,000 by 2,000 nautical miles. The hexagonal overlay design is used to provide an efficient means to calculate and model force movement and to describe both terrain and man-made obstacles. Each hexagon, in the database, is described in terms of its relative geographic location, the terrain within the hexagon boundaries, the elevation, and the barriers on each of the six sides. Hexagon size and the number of hexagons represented in a terrain database are user-data entries. Locations of objects in the game can be displayed as a hexagonal reference, latitude/longitude, or a military grid reference. Objects can be located anywhere on the game surface and are not limited to the center of the hexagons. 
JTLS does not require programming knowledge to use it effectively. As an interactive model, it requires human decisions to manage the processes and entities. The players receive messages and reports concerning the movement, attrition, and logistics status of their own forces, as well as intelligence summaries and capabilities of opposing forces. The player at each workstation can elect to view messages in plain language or a special military format. Messages may be electronically sent to standard Simple Message Text Protocol (SMTP) electronic mail workstations. Electronic feeds to several military command and control systems, such as the Global Command Control System, Joint Operational Tracking System, and Joint Military Command Information System, have been demonstrated.

The players interact with JTLS and receive graphical feedback through the Graphics Input Aggregate Control (GIAC). They receive messages through the Message Processor Program (MPP), and status board information is presented by the Information Management Terminal (IMT). These programs obtain their data and communicate with the main simulation component, the Combat Events Program, through software modules called the G Data System, using its data server program, GENIS. A single GENIS (the primary GENIS) is connected to the Combat Events Program using the TCP/IP network protocol. A GENIS may have other GENISes or interface programs as clients. The number of clients that a single GENIS can have at one time is determined by a system parameter of the machine on which it is executing. The parameter defaults to 64 on most machines, and can be modified by system maintenance personnel. A typical player's workstation has a GIAC, MPP and IMT, all operating and connected to a GENIS.

JTLS can be operated on a single workstation, or multiple workstations, and distributed on either a Local Area Network (LAN) or a Wide Area Network (WAN), thus providing a distributed exercise/gaming environment. The computer system support requirements for conducting simulations or analytic excursions using the JTLS model are dependent on the specifics of the event. The purpose of one event can be quite different from another (e.g., analysis, education, contingency plan development, etc.), and could require different support systems. The computing system is a composite of resources such as hardware devices, system software and utilities, communication lines, language compilers and databases.

The JTLS system can be run on a workstation of very limited processing power. For very small test databases, the CEP, GENIS, and two player suites (controller and one side) can be run on a single workstation of the SPARC station 2 class, but system performance is marginal. For exercise applications, in general, each active player requires a workstation of at least SPARC station 5 capability with 32 megabytes (MB) of random access memory (RAM) to perform adequately. For medium-size databases, the CEP and the primary GENIS each should have a SPARC station 20 
level workstation with $128 \mathrm{MB}$ of RAM, and each subordinate GENIS should have a workstation of at least SPARC station 20 level processing power, with $64 \mathrm{MB}$ of RAM.

The JTLS source, object, and executable files occupy approximately $550 \mathrm{MB}$ of disk storage. A medium to large database might require another $50 \mathrm{MB}$ of storage. Each checkpoint will use between two and four times as much storage as the initial database, depending largely on the intensity with which player's messages are managed. A 1.3-gigabyte disk devoted to the game directory (with tape backup) is a reasonable starting requirement.

Most of the JTLS system is written in the SIMSCRIPT II.5 programming language. It continuously improves with new technologies. Los Alamos National Laboratories has developed a graphics user-interface. ROLANDS \& ASSOCIATES Corporation $(\mathrm{R} \& \mathrm{~A})^{20}$ and NC3A have created several tools for the development of scenarios for JTLS. JTLS has been successfully used in conjunction with live training during exercises. For example, the KEEN EDGE (U.S. - Japanese) and COBRA GOLD (U. S. - Thailand) annual exercises both included live training. KEEN EDGE 95 was held at Camp Ojojihara, Japan to introduce U.S. and Japanese Ground Self-Defense Force armed forces and civilian counterparts to each other's way of doing business. COBRA GOLD is a joint exercise with the U.S. and Thailand Forces held in Thailand.

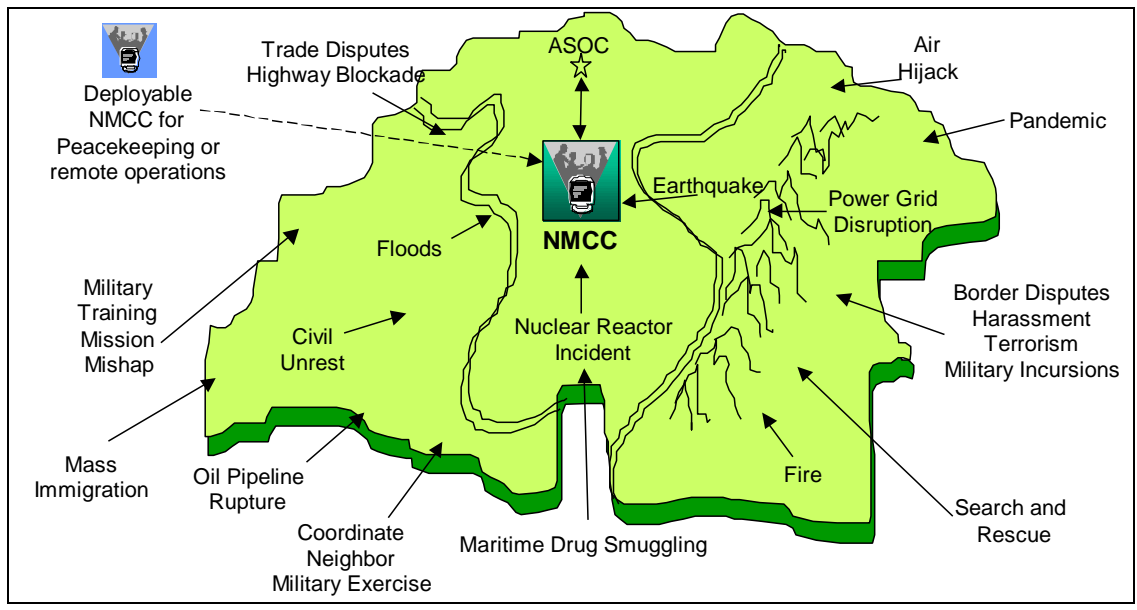

Figure 1. NMCC and JTLS Support to Crisis Management Operations 


\section{Future Topics}

The NMCC is visualized as a centralized facility to provide national-level coordinated management for military and civil crisis response. It will be initially controlled and operated by the MoD, with civil agency participation/liaisons. The NMCC concept includes interfaces with service headquarters, national military information sources, national civilian agencies/organizations, and regional or foreign agencies/organizations to receive and disseminate information. Figure 1 shows different scenarios in which the NMCC and JTLS might provide support to model and/or manage crisis response operations.

The next piece in this series will focus on the synergism between the potential capabilities of the NMCC and the inherent features contained in the current release of the Joint Theater Level Simulation (JTLS) software. Future topics planned for this series will include discussions on security issues relative to this concept, the future of JTLS within the U.S. M\&S community, NATO M\&S Policy relative to JTLS and current uses of the JTLS software in various colleges and universities.

1. National Military Command Center Concept of Operations, 22 November 1999 (draft).

2. The Joint Theater Level Simulation, presented by Dr. R. J. Roland and demonstrated by Mr. R. Kalinyak, International C4/NMCC Conference, 21-23 June 1999, Sofia, Bulgaria.

3. Keebom Kang and Ronald J. Roland, "Chapter 19. Military Simulation," in Handbook of Simulation: Principles, Methodology, Advances, Applications, and Practice, ed. Jerry Banks (Co-published by Engineering \& Management Press and John Wiley \& Sons, Inc., 1998), 645-658.

4. Please note that in the context of this paper, all references to military or government organizations will be U.S. unless otherwise noted.

5. It can be downloaded from http://www.dmso.mil/docslib/mspolicy/glossary/glossary1195.pdf.

6. Additional details about HLA can be found at http://hla.dmso.mil.

7. See also http://www.dmso.mil/wrkgrps/excims/charter.txt.

8. See http://www.amso.army.mil.

9. See http://www.amso.army.mil/amso2/armymsrr/

10. http://www-nsc.army.mil

11. See http://hp01.arc.iquest.com/mosaic/mosaic.html

12. For more information regarding the organizations and groups involved in military $M \& S$ activities see http://www.dmso.mil/orgs.html\#OEGS.

13. See http://www-nsc.army.mil/

14. For an example, see the internet homepage http://www.bgm.link.com/mfs.html for different military flight simulators.

15. Published by Monch Publishing Group, Federal Republic of Germany, ISSN 0937-6348. 
16. See http://www.rolands.com/jtls.html.

17. A History of the JTLS Development is available from ROLANDS \& ASSOCIATES Corporation.

18. Kang and Roland, "Military Simulation," discusses JTLS for military operations other than war.

19. Kevin Brandt and Ellen Roland, "Modeling Coalition Warfare: A Multi-Sided Simulation Design," in WSC 93 PROCEEDINGS: WINTER SIMULATION CONFERENCE PROCEEDINGS 1993, ed. Gerald W. Evans, Mansooreh Mollaghasemi, Edward C. Russell and William E. Biles (Winter Simulation Board of Directors, 1993. Library of Congress Number 87-654182), 977-983.

20. See http://www.rolands.com.

RONALD J. ROLAND, Ph.D., Management, University of Nebraska, Lincoln, NE, 1980 (dissertation on Using Artificial Intelligence Techniques to Design and Evaluate Decision Support Systems); M.S., Computer Science, University of Hawaii, Honolulu, HI, 1971; B.S., Mathematics, Colorado State University, Fort Collins, CO, 1969. Dr. Roland is the co-founder and President ROLANDS \& ASSOCIATES CORPORATION (R\&A). He has managed the development of several computer simulations including the Joint Theater Level Simulation (JTLS), Planning Alternatives for Interdicting National Terrorism (PAINT), and the Advanced Land Air Research Model (ALARM). JTLS is distributed and supported on a worldwide basis by R\&A through a CRADA. It is used for command post exercise support, analyses of contingency and operational plans, and examination of potential conflict situations. PAINT was used to develop counter terrorism plans, and ALARM was a research project designed to investigate organization structures, communications flows and movement of combat forces. Dr. Roland is Project Manager of the Naval Postgraduate School's (NPS) Academic and Research support contract and the CINCPACFLT Wargaming Division support contract. He is also the R\&A corporate pilot.

Dr. Roland was a Professor in the Computer Science Department of the NPS where he designed and presented courses in computer science, command control, and information systems. He conducted research in artificial intelligence and advanced technology for management decision support systems. Dr. Roland acted as the project manager and director for acquisition, installation, and implementation of their first modeling, simulation and wargaming laboratory. His publications are available on request. Additional information is available concerning R\&A at www.rolands.com. E-mail address: President@ rolands.com. 